'Departamento de HematologíaOncología, Facultad de Medicina, Pontificia Universidad Católica de Chile. Santiago, Chile. ${ }^{2}$ Servicio de Oncología, Hospital Base San José. Osorno, Chile.

${ }^{3}$ Departamento de Endocrinología, Facultad de Medicina, Pontificia Universidad Católica de Chile. Santiago, Chile.

${ }^{4}$ Departamento de Endocrinología, Hospital las Higueras. Talcahuano, Chile. ${ }^{5}$ Departamento de Radiología, Facultad de Medicina, Pontificia Universidad Católica de Chile. Santiago, Chile. ${ }^{6}$ Departamento de Anatomía Patológica, Facultad de Medicina, Pontificia Universidad Católica de Chile. Santiago, Chile.

${ }^{7}$ Servicio de Oncología Médica, Fundación Arturo López Pérez. Santiago, Chile. aPhD.

Trabajo no recibió financiamiento. Los autores declaran no tener conflictos de interés.

Recibido el 1 de junio de 2020, aceptado el 13 de enero de 2021

Correspondencia a: Dr. Marcelo Garrido Departamento de HematologíaOncología, Facultad de Medicina, Pontificia Universidad Católica de Chile. Santiago, Chile. mgarrido@med.puc.cl

\section{Tumores Neuroendocrinos: un desafío transversal}

\author{
DIEGO CARRILLO ${ }^{1}$, MATÍAS MUÑOZ-MEDEL ${ }^{1}$, \\ IGNACIO RETAMAL ${ }^{1, a}$, MAURICIO PINTO ${ }^{1, a}$, \\ MARÍA L. BRAVO ${ }^{1, a}$, BRUNO NERVI ${ }^{1}$, JOSÉ PEÑA ${ }^{1}$, \\ YASNA VALENZUELA ${ }^{2}$, FRANCISCO J. GUARDA ${ }^{3}$, \\ FLAVIA NILO ${ }^{3}$, FERNANDO BELLO $^{4}$, PILAR ORELLANA ${ }^{5}$, \\ DANIEL VICENTINI ${ }^{5}$, JUAN CARLOS QUINTANA ${ }^{5}$, \\ PAULA J. TORRES ${ }^{6}$, JOSÉ LUIS LEAL ${ }^{7}$, MARCELO GARRIDO ${ }^{1}$
}

\section{Update on neuroendocrine tumors}

Neuroendocrine Tumors (NETs) encompass a wide variety of tumors arising from neuroendocrine cells, which produce bioactive substances. The incidence of NETs increased significantly lately, becoming one of the most common tumors of the digestive tract. Their clinical presentation is as diverse as their capacity for hormone production. Carcinoid syndrome is the most common hormonal syndrome produced by NETs and is characterized by diarrhea, flushing and cardiac valvular lesions. New research brought multiple changes in the classification of these neoplasms and a new understanding about their diagnosis and treatment, promoting a multidisciplinary approach. Somatostatin analogues, radiation, biological, and cytotoxic drugs have improved the prognosis of these patients, which entails a great challenge for healthcare providers.

(Rev Med Chile 2021; 149: 888-898)

Key words: Biomarkers, Tumor; Malignant Carcinoid Syndrome; Neuroendocrine Tumors; Somatostatin.

\section{L} os tumores neuroendocrinos (TNEs) constituyen un grupo heterogéneo de neoplasias que se originan a partir de células neuroendocrinas, productoras de monoaminas, péptidos y otras sustancias biológicamente activas, ampliamente distribuidas en el organismo. El estudio de los TNEs tiene más de un siglo de historia. En 1888, Lubarsch fue uno de los pioneros al describir múltiples tumores en el íleon en una autopsia ${ }^{1}$; poco después, en 1907, Oberndorfer acuñó el término karzinoid para describir un grupo de tumores con un comportamiento menos agresivo comparado con los adenocarcinomas ${ }^{2}$. En la década de 1950 surgieron los primeros reportes de manifestaciones clínicas características asociadas a tumores carcinoides, lo que posteriormente se denominó sindrome carcinoide (SC).

\section{Epidemiología}

La incidencia de los TNEs ha aumentado progresivamente durante las últimas décadas ${ }^{3}$, desde 1,09 casos por 100.000 habitantes en 1975 hasta alcanzar 6,98 casos por 100.000 en 2012 en Estados Unidos. Este aumento se debe, principalmente, a tumores de bajo grado, en estadios tempranos, y a edades avanzadas ${ }^{4}$. Se cree que los avances en las técnicas de diagnóstico y los cambios demográficos de la población podrían estar involucrados.

La evolución indolente de un grupo de TNEs y la mejora del pronóstico dada por los nuevos tratamientos, han traído como consecuencia una alta prevalencia de esta enfermedad; actualmente, los TNEs ocuparían el segundo lugar en prevalencia dentro de las neoplasias digestivas en Estados 
Unidos, solo detrás del cáncer colorrectal ${ }^{5}$. En Chile, no se conoce con precisión la incidencia ni prevalencia de los TNEs. En 2015 se implementó el primer registro multicéntrico de TNEs en el país, cuyo primer reporte se publicó en 2018, con 166 pacientes de 6 centros oncológicos del país. La edad promedio de presentación fue de 53 años, $64 \%$ fueron TNEs digestivos y $62 \%$ debutó en etapa $\mathrm{IV}^{6}$.

La mayoría de los TNEs se presentan de forma esporádica, mientras que 15 a 20\% están asociados a síndromes genéticos que predisponen a presentar TNEs, como Neoplasia Endocrina Múltiple tipo 1 y 2 (NEM1 y NEM2), Von Hippel-Lindau, Neurofibromatosis tipo 1 y Esclerosis Tuberosa ${ }^{7}$.

\section{Histología}

En la microscopía óptica, los TNEs se presentan con células de citoplasma anfófilo y núcleos redondeados, con cromatina granular (patrón "en sal y pimienta"). En la inmunohistoquímica (IHQ), se caracterizan por expresar marcadores citoplasmáticos de diferenciación neuroendocrina, donde los principales son la Cromogranina A (CgA) y la Sinaptofisina. Otros marcadores IHQ utilizados son el CD56 y Enolasa Específica Neuronal (NSE), aunque estos últimos de baja especificidad para diferenciación neuroendocrina. Para evaluar su índice proliferativo se utiliza el número de mitosis en $2 \mathrm{~mm}^{2}$ (evaluando al menos $10 \mathrm{~mm}$ cuadrados de tumor) y el índice de proliferación celular medido con Ki-67, con un conteo mínimo de 500 células tumorales. En ambos casos deben seleccionarse las áreas de mayor proliferación ${ }^{8}$.

Los TNEs bien diferenciados comparten más características con el tejido neuroendocrino normal y pueden tener tasas de proliferación celular variadas. En contraste, los TNEs poco diferen- ciados, tradicionalmente llamados carcinomas neuroendocrinos (CNE), presentan una menor expresión de marcadores neuroendocrinos y tasas de proliferación siempre elevadas. Los CNE, además, pueden encontrarse en asociación con neoplasias exocrinas, como el adenocarcinoma, lo que es extremadamente infrecuente en TNEs bien diferenciados?.

\section{Clasificación}

Históricamente, la nomenclatura y clasificación de los TNEs ha sido confusa y controvertida, y se basa en el sitio del tumor primario, origen embriológico, grado histológico, y estado funcional ${ }^{8}$.

Una de las clasificaciones comúnmente aceptada es la de la Sociedad Europea de Tumores Neuroendocrinos (ENETS) para los TNEs gastroenteropancreáticos bien diferenciados, agrupándolos según su grado histológico $(1,2 \text { o } 3)^{10}$ (Tabla 1$)$. La Organización Mundial de la Salud (OMS), a su vez, ha propuesto diversas clasificaciones histológicas con diferencias sustanciales entre sí. La más reciente de ellas, de finales de 2017, agrupa a los TNEs pancreáticos en 3 categorías: bien diferenciadas (TNE grado 1, 2 o 3), pobremente diferenciadas (CNE grado 3 de células grandes o pequeñas) y Neoplasia Mixta Neuroendocrina No Neuroendocrina o MiNEN (Mixed Neuroendocrine-Non-neuroendocrine Neoplasm) ${ }^{11}$ (Tabla 2). Ambas clasificaciones utilizan la proporción de Ki-67 en la muestra y el índice mitótico como estimaciones de la proliferación.

La American Joint Committee on Cancer (AJCC) y la ENETS han propuesto sistemas de etapificación según TNM conceptualmente similares, pero con algunas diferencias que podrían tener implicancias en la estimación del pronóstico ${ }^{12}$, por lo que es indispensable precisar el sistema utilizado.

Tabla 1. Clasificación del grado histológico de TNEs Gastroenteropancreáticos bien diferenciados según la ENETS 2014

\begin{tabular}{|ccc|}
\hline Grado histológico & Mitosis & Ki $\mathbf{6 7}$ \\
\hline Bajo (grado 1) & 0 a 1 por 10 campos & Menor a $3 \%$ \\
\hline Intermedio (grado 2) & 2 a 20 por 10 campos & 3 a $20 \%$ \\
\hline Alto (grado 3) & Más de 20 por 10 campos & Mayor a $20 \%$ \\
\hline
\end{tabular}


Tabla 2. Clasificación histológica de TNEs de páncreas endocrino según la OMS 2017

\begin{tabular}{|llll|}
\hline Grado histológico & & Mitosis & Ki 67 \\
$\begin{array}{l}\text { Neoplasia neuroendocrina bien } \\
\text { diferenciada }\end{array}$ & TNE grado 1 & 0 a 1 por 10 campos & Menor a 3\% \\
& TNE grado 2 & 2 a 20 por 10 campos & 3 a 20\% \\
TNE grado 3 & Más de 20 por 10 campos & Mayor a 20\% \\
Neoplasia neuroendocrina & CNE grado 3 (de células grandes o & Más de 20 por 10 campos & Mayor a 20\% \\
\hline
\end{tabular}

Neoplasia mixta neuroendocrina - no neuroendocrina (MiNEN).

Finalmente, la producción de sustancias con actividad biológica puede ser utilizada para clasificar a los TNEs en funcionantes y no funcionantes. Esto se determina según la presencia de síntomas producto de la secreción de hormonas y la confirmación por exámenes de laboratorio del exceso de sustancias derivadas de la hipersecreción hormonal ${ }^{13}$.

\section{Presentación clínica}

Los TNEs pueden debutar de forma incidental, por síntomas producidos por efecto de masa o por enfermedad avanzada ${ }^{14}$, y en el caso de TNEs funcionantes, asociados a diversos síndromes clínicos derivados de la hiperproducción hormonal. El SC es el más frecuente, presente en 19\% de los TNEs al momento del diagnóstico ${ }^{15}$. Este se relaciona con la secreción de sustancias como serotonina, calicreína, histamina, bradicininas y taquiquininas, entre otras, mayoritariamente en TNEs bien diferenciados de origen intestinal, llamados clásicamente tumores carcinoides ${ }^{16}$. Una vez que se presentan metástasis hepáticas, las sustancias producidas pueden evitar su degradación en el hígado y llegar directamente a la circulación sistémica, provocando los síntomas característicos del SC: bochornos, diarrea, dolor abdominal, obstrucción bronquial y daño valvular cardiaco. Si existe la sospecha clínica de SC, el estudio debe incluir una ecocardiografía para evaluar el compromiso cardiaco ${ }^{17}$.

Otros síndromes pueden presentarse en TNEs funcionantes, como insulinomas, gastrinomas, feocromocitomas o hipercortisolismo, entre otros, dependiendo de la hormona específica secretada (Tabla 3).

\section{Estudio de imágenes}

La tomografía computada (TC) con medio de contraste yodado entrega una adecuada caracterización en la mayoría de los casos; los tumores primarios y las metástasis se presentan frecuentemente como lesiones sólidas hipervasculares, ocasionalmente con componente quístico, y en algunos casos, con un patrón de realce hipovascu$\operatorname{lar}^{18}$ (Figura 1B). La resonancia magnética (RM) logra un mayor contraste intrínseco, con una mejor sensibilidad y especificidad, especialmente en TNEs hepáticos y pancreáticos.

Durante las últimas décadas se han desarrollado métodos de imágenes funcionales que aprovechan la sobreexpresión de receptores de somatostatina en TNEs bien diferenciados. Estas técnicas son utilizadas en el estudio diagnóstico, etapificación, seguimiento y evaluación de respuesta a tratamiento en TNEs bien diferenciados. La cintigrafía planar, en 2 dimensiones, y SPECT, en 3 dimensiones, utilizan análogos de somatostatina marcados con emisores gamma. La Tomografía por Emisión de Positrones-Tomografía Computada (PET/CT) utiliza análogos de somatostatina marcados un emisor de positrones denominado Galio ${ }^{68}$, como el Ga ${ }^{68}$ DOTA-TOC (DOTA-D-Phe1-Tyr3-Octreotide), $\mathrm{Ga}^{68}$-DOTA-NOC (DOTA-1-Nal3-Octreotide) y $\mathrm{Ga}^{68}$-DOTA-TATE (DOTA-D-Phe1-Tyr3-Thr8Octreotide). Tanto la cintigrafía como el PET/CT con análogos de somatostatina son útiles en TNEs bien diferenciados, pero el PET/CT tiene mejor rendimiento en la clínica ${ }^{19}$ (Figura 2B).

Por otra parte, los CNE y algunos TNEs de alto grado presentan una menor expresión de receptores de somatostatina y una mayor actividad metabólica, por lo que el estudio de imágenes 
Tabla 3. Principales síndromes asociados a TNE funcionantes y sus características clínicas

\begin{tabular}{|c|c|c|c|}
\hline $\begin{array}{l}\text { Tipo de TNE } \\
\text { funcionante }\end{array}$ & $\begin{array}{l}\text { Principal hormona } \\
\text { secretada }\end{array}$ & $\begin{array}{l}\text { Principal } \\
\text { síndrome }\end{array}$ & Manifestaciones clínicas \\
\hline Tumor carcinoide & Serotonina & Carcinoide & $\begin{array}{l}\text { Diarrea, bochornos, dolor abdominal, valvulopatía tricus- } \\
\text { pidea, obstrucción bronquial }\end{array}$ \\
\hline Insulinoma & Insulina & Insulinoma & Hipoglicemia \\
\hline Gastrinoma & Gastrina & Zollinger-Ellison & Dolor abdominal, diarrea, enfermedad péptica \\
\hline $\begin{array}{l}\text { Tumor productor } \\
\text { de ACTH }\end{array}$ & ACTH & Cushing ectópico & $\begin{array}{l}\text { Hiperpigmentación de piel y mucosas, hiperglicemia, } \\
\text { hipertensión refractaria, hipokalemia, miopatía proximal, } \\
\text { inmunosupresión, tromboembolismo, equimosis fácil y } \\
\text { síndrome hemorragíparo }\end{array}$ \\
\hline Feocromocitoma & $\begin{array}{l}\text { Adrenalina, } \\
\text { noradrenalina }\end{array}$ & Hiperadrenérgico & Hipertensión, sudoración, cefalea, palidez, taquicardia \\
\hline VIPoma & $\begin{array}{l}\text { Péptido vasoactivo } \\
\text { intestinal }\end{array}$ & $\begin{array}{l}\text { Verner-Morrison, } \\
\text { cólera pancreático }\end{array}$ & $\begin{array}{l}\text { Diarrea acuosa, deshidratación, hipokalemia, aclorhidria, } \\
\text { hipercalcemia }\end{array}$ \\
\hline Glucagonoma & Glucagón & Glucagonoma & $\begin{array}{l}\text { Hiperglicemia, baja de peso, diarrea, tromboembolismo, } \\
\text { eritema necrolítico migratorio }\end{array}$ \\
\hline Somatostatinoma & Somatostatina & Somatostatinoma & $\begin{array}{l}\text { Diabetes, diarrea, esteatorrea, patología biliar, aclorhi- } \\
\text { dria, baja de peso }\end{array}$ \\
\hline
\end{tabular}

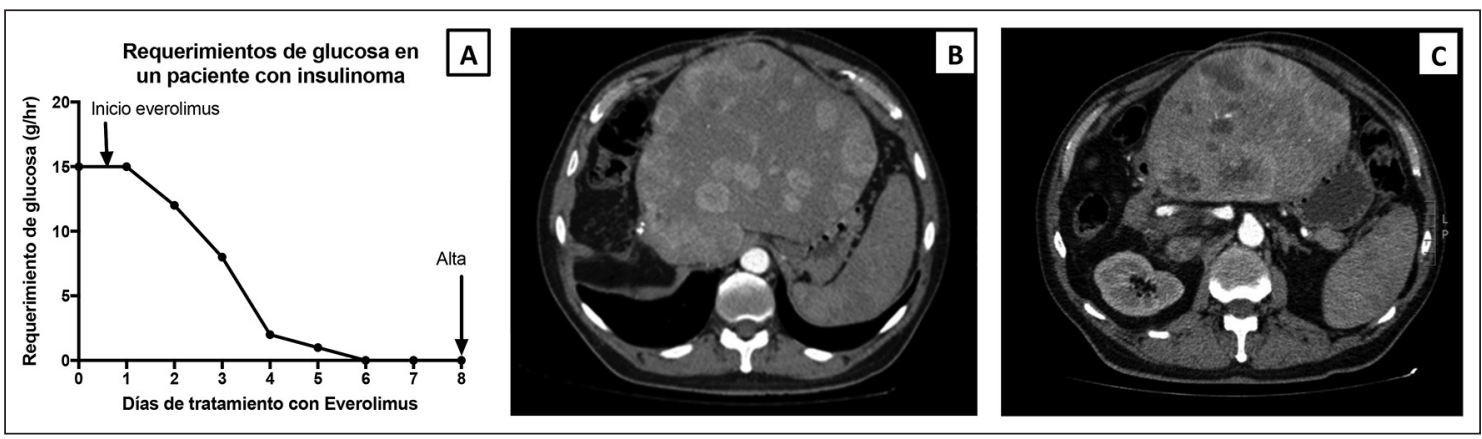

Figura 1. Imágenes seleccionadas correspondientes a un paciente de 54 años con diagnóstico de insulinoma metastásico, hospitalizado en una Unidad de Pacientes Críticos por hipoglicemias de difícil manejo. (A) Disminución progresiva de los requerimientos de aporte parenteral de glucosa desde el inicio de terapia con Everolimus. (B) Corte axial de TC en fase arterial que muestra extenso compromiso hepático secundario. (C) Disminución del compromiso hepático luego del tratamiento con Everolimus.

funcionales de elección en estos casos es el PET-CT con ${ }^{18} \mathrm{FDG}$ (Fluorodeoxiglucosa) ${ }^{18}$.

\section{Biomarcadores}

Los TNEs constituyen un espectro heterogéneo de enfermedades, por lo que no existe un marcador ideal para su diagnóstico. Uno de los más utilizados es la CgA, una proteína de la familia de las graninas secretada por células neuroendocrinas en conjunto con otras hormonas y neurotransmisores, cuya secreción puede persistir aún cuando la célula neuroendocrina sufre una transformación neoplásica ${ }^{20}$. Actualmente, $\mathrm{CgA}$ es el biomarcador más estudiado y con el mejor rendimiento en la clínica $^{21}$. Diversos estudios han correlacionado sus niveles plasmáticos con la carga tumoral y la sobrevida. La sensibilidad de la CgA fluctúa entre $43-100 \%$ y su especificidad entre $10-96 \%{ }^{21}$, de- 

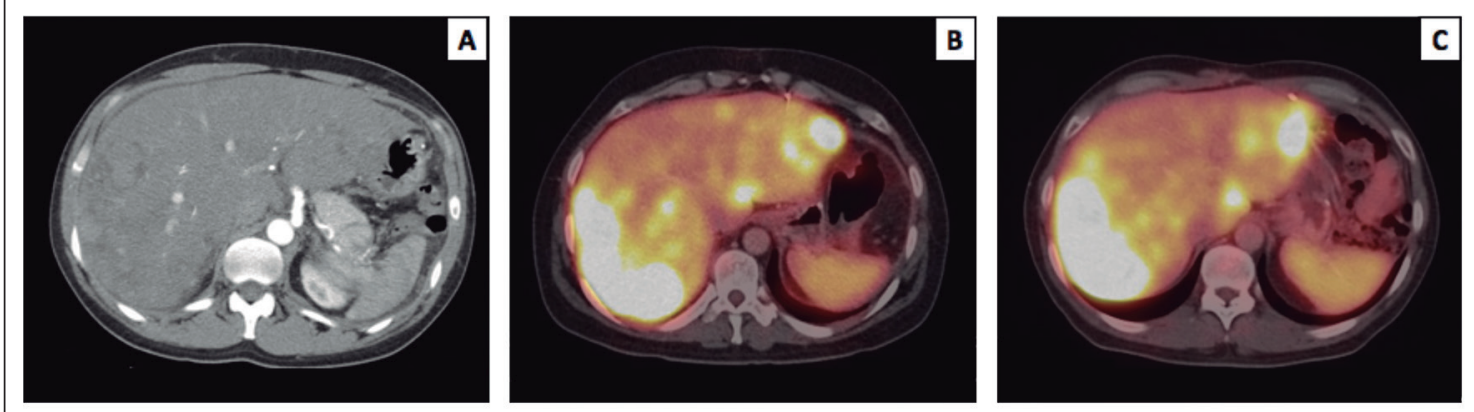

Figura 2. Imágenes seleccionadas de un paciente de 33 años que consulta por síndrome carcinoide. (A) Corte axial de TC en fase arterial muestra una lesión hipervascular en la cola del páncreas, compatible con un tumor neuroendocrino, asociado a múltiples lesiones hepáticas secundarias. (B) Corte axial de PET ${ }^{68} \mathrm{Ga}$-DOTATATE luego de haber progresado al tratamiento con análogos de somatostatina. Se observa intensa captación del marcador a nivel hepático, dado por extenso compromiso secundario de tumor neuroendocrino. (C) Corte axial de PET-68Ga-DOTATATE luego de haber recibido 4 dosis de tratamiento de segunda línea con el radiofármaco ${ }^{177}$ Lu-DOTATATE. Se observa estabilidad del tamaño de las lesiones hepáticas, pero menor intensidad de la captación del marcador, compatible con respuesta oncológica parcial. Clínicamente, este hallazgo se correlacionó con disminución síntomas de hipersecreción hormonal y de biomarcadores como CgA y Ácido 5-HIIA.

pendiendo del origen, carga tumoral e histología. Los casos de falsos positivos pueden deberse a insuficiencia renal, fármacos como los inhibidores de la bomba de protones, algunas endocrinopatías, enfermedades inflamatorias y algunos tipos de cáncer no TNE, entre otros ${ }^{22}$.

La serotonina intraplaquetaria (SIP), y su producto de degradación, el ácido 5-Hidroxiindolacético (5-HIIA ${ }^{23}$, cuantificable en orina, son altamente específicos (hasta 100\%) para tumores carcinoides, y se puede relacionar con presencia de SC, sin embargo, su sensibilidad es tan solo de $35 \%$ para TNEs, llegando hasta $75 \%$ en tumores bien diferenciados ${ }^{24}$ (Figura 3).

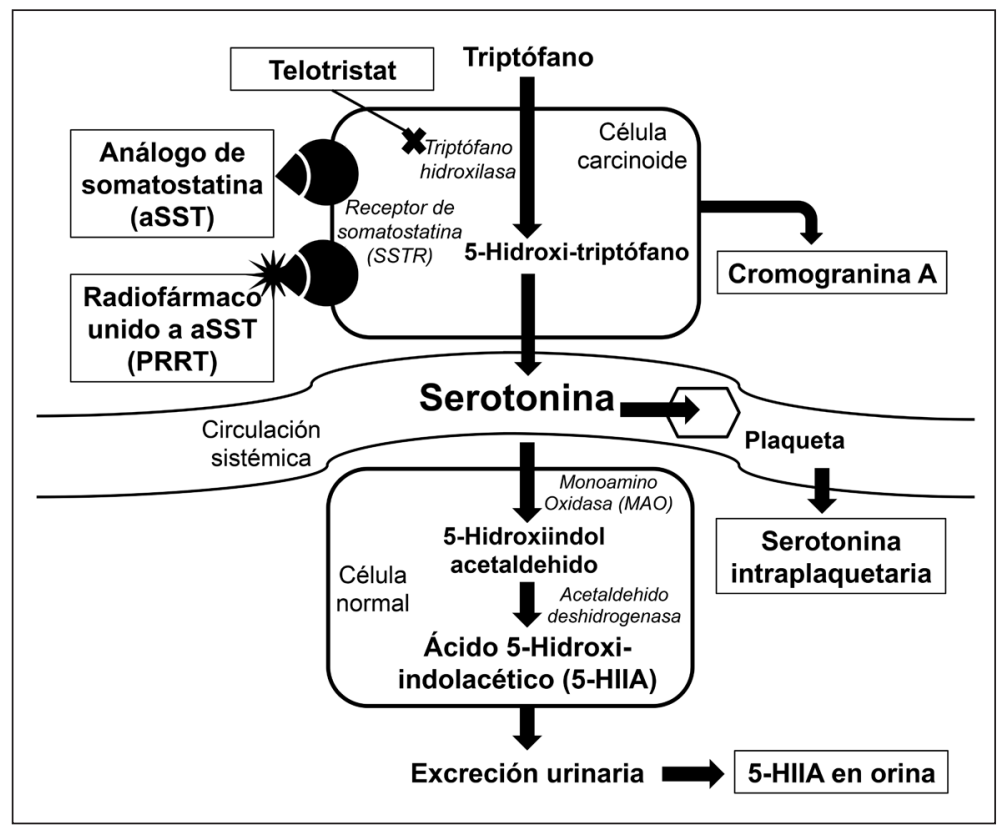

Figura 3. Esquema del modelo fisiopatológico del tratamiento con aSST y telotristat. Biomarcadores en TNEs. 
Según la sospecha clínica de funcionalidad se pueden medir hormonas específicas secretadas por el tumor, como gastrina, glucagón, insulina, catecolaminas, metanefrinas, y cortisol, con un alto rendimiento. Sin embargo, estos métodos son solo aplicables para el grupo minoritario de TNEs funcionantes ${ }^{25}$.

Existe además una serie de nuevos biomarcadores potencialmente útiles para TNEs, incluyendo células tumorales circulantes ${ }^{26}$, marcadores genómicos ${ }^{27}$, metabolómicos ${ }^{28} \mathrm{y}$ análisis por multimarcadores ${ }^{29}$, actualmente en proceso de validación clínica.

\section{Tratamiento}

El manejo de los TNEs se orienta principalmente al control oncológico y de los síntomas producidos por la hipersecreción hormonal, y varía desde la observación a la aplicación de terapias multimodales según cada paciente en particular. Dada la complejidad y las múltiples alternativas terapéuticas actualmente disponibles, el tratamiento debe ser definido y realizado por equipos multidisciplinarios, en los que participen especialistas en endocrinología, cirugía, radioterapia, oncología médica, anatomía patológica, radiología intervencionista, medicina nuclear y cardiología ${ }^{30}$. La elección de la terapia sistémica se basa en el grado de diferenciación, origen y compromiso clínico; la secuencia y combinación en la que se realizan estos tratamientos debe ser evaluada caso a caso. En algunos pacientes con TNE bien diferenciados avanzados, asintomáticos y sin progresión evidente, podría plantearse el seguimiento (Figura 4 ).

\section{Tratamientos locales}

En TNEs localizados y localmente avanzados, la cirugía es el tratamiento de elección, ya que logra altas tasas de curación en etapas precoces de la enfermedad. En TNEs pancreáticos, la sobrevida libre de recurrencia a 5 años alcanza 100\%, 70\% y $53 \%$, para estadios I, II y III de la ENETS ${ }^{31}$. La quimioterapia y radioterapia tienen un rol en el tratamiento definitivo del CNE localizado.

En pacientes con TNEs bien diferenciados

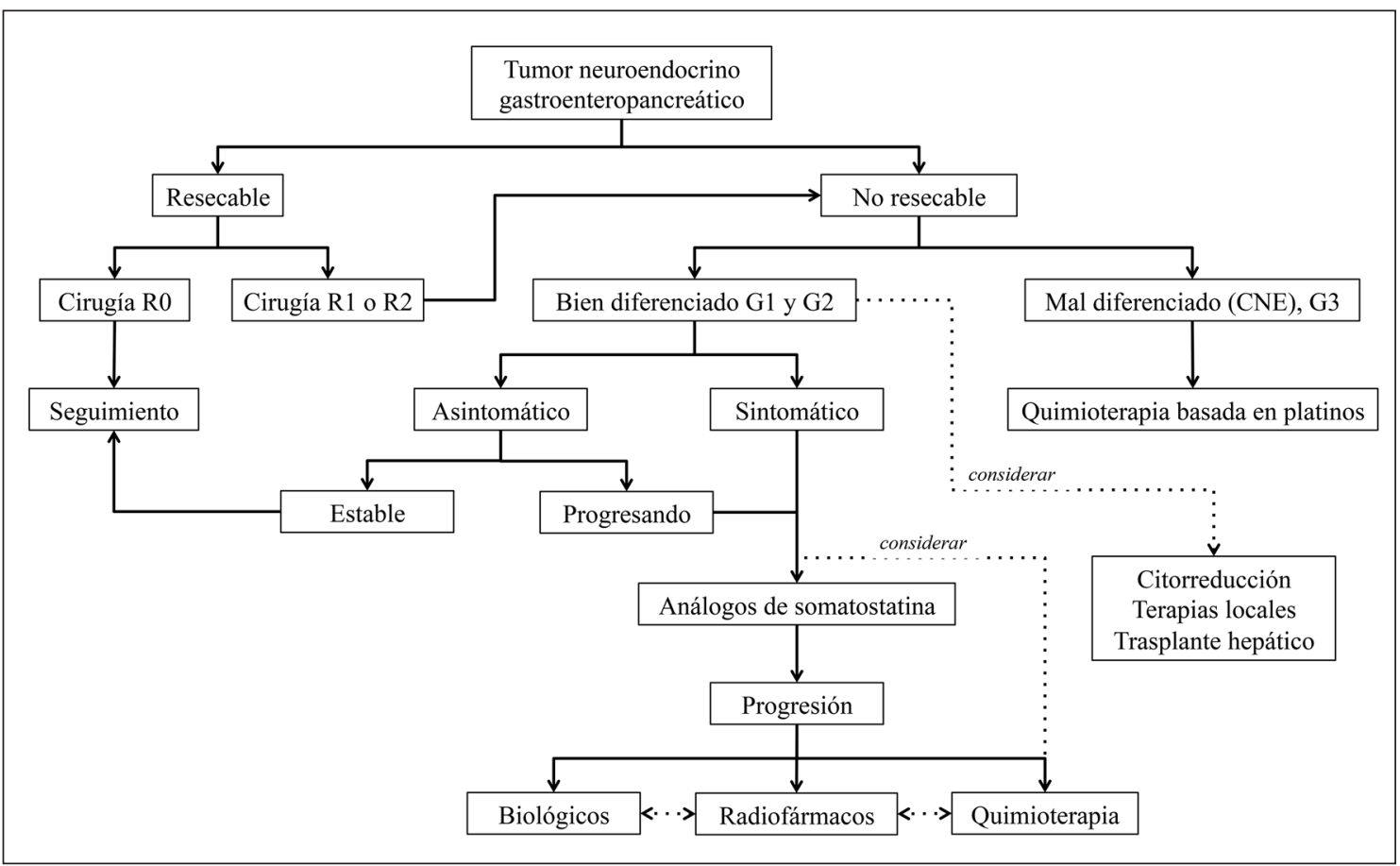

Figura 4. Algoritmo de manejo de TNEs Gastroenteropancreáticos. En el escenario de enfermedad no resecable, el tratamiento depende fundamentalmente de los síntomas, la histología del tumor y del origen pancreático versus extrapancreático. 
con enfermedad avanzada, la cirugía citorreductiva y los tratamientos dirigidos al hígado, como la ablación por radiofrecuencia, quimioembolización y radioterapia interna selectiva, tienen un beneficio en casos seleccionados. El trasplante hepático es una alternativa para el tratamiento de metástasis hepáticas exclusivas irresecables, en pacientes menores de 55 años, previa resección del tumor primario, con enfermedad estable 6 meses previo al trasplante, Ki 67 positivo menor a $10 \%$, y compromiso tumoral menor a $50 \%$ del hígado ${ }^{32}$. Menos de 1\% de los pacientes con enfermedad avanzada son candidatos a esta terapia.

\section{Análogos de somatostatina (aSST)}

Se utilizan para el control de síntomas en TNEs bien diferenciados en estadios avanzados, sin embargo, recientemente se han reconocido sus efectos antitumorales y su beneficio en sobrevida libre de progresión $(\mathrm{SLP})^{33}$. El primero en desarrollarse fue el octreótide ${ }^{34}$ que requería múltiples dosis diarias. Posteriormente aparecieron fórmulas de acción prolongada como el octreótide $\mathrm{LAR}^{35}$, lanreóti$\mathrm{de}^{36} \mathrm{y}$ pasireótide ${ }^{37}$. El estudio fase III PROMID, mostró una SLP significativamente mayor en el grupo tratado con octeótride LAR versus placebo (14,3 versus 6 meses) en TNEs bien diferenciados de intestino medio metastásicos. Asimismo, el estudio fase III CLARINET obtuvo una mayor SLP en pacientes tratados con lanreótide versus placebo (media no alcanzada versus 18 meses) en pacientes con TNEs enteropancreáticos bien diferenciados no funcionantes. Algunos mecanismos que podrían explicar el efecto antitumoral de los aSST son, por un lado, la acción antimitótica directa, que induce detención del ciclo celular y/o apoptosis; y por otro lado, la acción indirecta inhibiendo la liberación de factores de crecimiento como el IGF-1, la Insulina y la Gastrina, entre otros (Figura 3). En general, estos fármacos son bien tolerados, aunque su uso en pacientes con insulinoma debe ser controlado, pues pueden empeorar la hipoglicemia debido a la supresión del glucagón ${ }^{36}$.

\section{Radiofármacos (PRRT)}

La modalidad más estudiada es la terapia con péptidos radiactivos dirigidos a receptores de somatostatina (PRRT). El primero que se utilizó fue $\mathrm{In}^{111}$-Pentetreótide, con respuesta oncológica solamente en $8 \%$ de los pacientes debido a la baja penetración del electrón Auger emitido por el $\operatorname{In}^{111}$ ${ }^{38}$. Actualmente se utilizan radioisótopos emisores beta de mayor energía (Lutecio-177 $\left(\mathrm{Lu}^{177}\right)$ o Itrio-90 $\left(\mathrm{Y}^{90}\right)$ ), asociados a análogos sintéticos de somatostatina (generalmente DOTATATE o DOTATOC), los que se unen y se depositan en células que expresan el receptor de somatostatina, otorgando un efecto de radiación localizada sobre las células tumorales (Figura 3). El estudio fase III NETTER-1 mostró una mayor SLP a 20 meses $(62,5$ vs $10,8 \%)$ y una mayor tasa de respuesta (18 vs $3 \%$ ) con PRRT (Lu ${ }^{177}$-DOTATATE) asociado a octreótide LAR versus octreótide LAR en forma aislada, en pacientes con TNE intestinales avanzados, progresando tras terapia de primera línea con aSST (Figura 2B y 2C). Los análisis preliminares muestran un aumento en la sobrevida global (SG), lo que deberá ser confirmado en el análisis final. Algunos efectos adversos graves como mielosupresión se presentaron en menos de $10 \%$ de los pacientes ${ }^{39}$. Esta terapia es particularmente promisoria en el tratamiento de los TNEs bien diferenciados. Otra estrategia en desarrollo es el uso de emisores alfa unidos a análogos de somatostatina $\left(\mathrm{Pb}^{212}\right.$-Octreotate, actualmente en estudio $)^{40}$.

\section{Terapias biológicas}

Se han utilizado diferentes vías de señalización celular para lograr efectos antitumorales en TNE. Algunas de las vías utilizadas son las Tirosina Kinasas (TK), "Mammalian Target of Rapacycin" (mTOR) y la del factor de crecimiento vascular endotelial (VEGF). El estudio fase III RADIANT-2 concluyó que Everolimus, un inhibidor de mTOR, mejoró la SLP frente a placebo $(16,4$ versus 11,3 meses) en TNEs bien diferenciados, funcionantes, provenientes del pulmón o enteropancreáticos tratados previamente con aSST ${ }^{41}$. Se han obtenido resultados similares en TNEs pancreáticos (RADIANT-3) ${ }^{42}$ y TNEs intestinales no funcionantes (RADIANT-4) ${ }^{43}$ (Figura 1). Sunitinib, un inhibidor de TK antiangiogénico demostró un aumento en SLP ( 11 vs 5,5 meses) y SG (92 vs $85 \%$ a 6 meses) en TNEs pancreáticos avanzados, bien diferenciados, en progresión ${ }^{44}$. Otros agentes biológicos, como el inhibidor del receptor del VEGF Bevacizumab, tienen un efecto al usarse en conjunto con otras terapias en escenarios seleccionados. 


\section{Quimioterapia citotóxica (QMT)}

Diversos agentes citotóxicos han sido utilizados en el tratamiento de los TNEs. La estreptozocina, un alquilante aprobado por la FDA para TNEs pancreáticos en el año 1982, actualmente se encuentra en desuso debido a sus efectos adversos. La temozolamida es un alquilante de administración oral que ha mostrado resultados favorables en estudios como monodroga o en combinación ${ }^{45,46}$. Otros regímenes basados en fluoropirimidinas como el 5-Fluouracilo ${ }^{47}$ o capecitabina ${ }^{46}$, en distintas combinaciones, han demostrado eficacia en TNEs digestivos. En TNEs bien diferenciados, la indicación de QMT se reserva para escenarios clínicos seleccionados: en el caso de tumores pancreáticos de moderado y alto grado (G2 y G3 de la ENETS), y en tumores extrapancreáticos de alto grado (G3 de la ENETS) ${ }^{48}$, se puede utilizar en pacientes con enfermedad rápidamente progresiva, sintomática y/o voluminosa. En CNE avanzado, la QMT basada en etopósido y platinos han demostrado entregar un beneficio significativo en sobrevida ${ }^{49}$, estableciéndose como la primera línea de tratamiento ${ }^{50}$. En este escenario, el índice de Ki67 $\geq 55 \%$ se relaciona con mejor respuesta a QMT con platinos ${ }^{51}$.

\section{Otras terapias dirigidas}

Existe una serie de fármacos utilizados para controlar los efectos de la hipersecreción hormonal, sin tener una acción antineoplásica conocida. El telotristat, de administración oral, es un inhibidor de la triptófano hidroxilasa, una enzima que transforma el triptófano a serotonina, y actúa disminuyendo la hipersecreción de serotonina en tumores carcinoides (Figura 3). Esta terapia dirigida, aprobada en 2017 por la FDA, ha demostrado utilidad en el manejo del SC refractario ${ }^{52}$. A su vez, los inhibidores de la bomba de protones (IBP), se utilizan para tratar la hipersecreción de ácido clorhídrico gástrico en el síndrome de Zollinger-Ellison secundario a gastrinoma. En el caso de los insulinomas, puede utilizarse el diazóxido, un activador de canales de potasio que inhibe la secreción de insulina, controlando los síntomas de hipoglicemia. En el Cushing Ectópico, el uso de inhibidores de la esteroidogénesis suprarrenal como el etomidato ${ }^{53}$ o ketoconazol, o antagonistas del receptor de glucocorticoides como la mifepristona, también han demostrado beneficio en hipercortisolismos graves ${ }^{54}$.

\section{Pronóstico}

La evolución y sobrevida de los pacientes con TNEs son muy variables. Los factores demográficos que clásicamente se han asociado a peor pronósticos son edad avanzada, sexo masculino, nivel socio-económico bajo y ruralidad ${ }^{51}$. Los relativos al tumor son el estadio avanzado, grado histológico y Ki67 positivo elevados ${ }^{55}$, tumores poco diferenciados, histología mixta con adenocarcinoma, y el origen colorrectal ${ }^{56}$ de la lesión primaria. Otras variables clínicas descritas son el mal estado funcional, la presencia de $S^{57}$ y biomarcadores, como la $\mathrm{CgA}$, plaquetas, $\mathrm{LDH}$, entre otros ${ }^{58}$. La SG media reportada en estudios poblacionales es de 75-112 meses $^{4,5}$, con una SG a 5 años de entre $56,5 \%$ y $75,4 \%{ }^{59,60}$. En Chile, la SG media reportada es de 110 meses, con una SG a 5 años de 91,3\% para los TNEs grado 1, 77,4\% para los grados 2 , y $60,1 \%$ para los grados $3^{6}$. En el caso de los CNE, el estudio escandinavo NORDIC NEC reporta una SG de 15 meses en tumores con Ki67 < 55\%, y 10 meses para Ki67 $\geq 55 \%{ }^{51}$. Estos hallazgos ponen en evidencia la importancia de la estimación de proliferación celular como marcador pronóstico en TNEs.

\section{Conclusión}

La complejidad biológica y la gran heterogeneidad que caracteriza a los TNEs, plantean múltiples desafíos en los ámbitos de diagnóstico, tratamiento, investigación traslacional y políticas públicas en salud. Los TNEs se perfilan como un modelo de enfermedad en el que cobra especial valor la interacción en equipos multidisciplinarios, la integración del sistema de salud en sus distintos niveles y la cooperación del mundo público, privado y la sociedad civil.

Durante los últimos años en Chile se ha mejorado el acceso a terapias específicas para TNEs, como los análogos de somatostatina o inhibidores de la vía mTOR, a través de protocolos de acceso a "medicamentos de alto costo", sin embargo, aún hay terapias para que no se encuentran disponibles en el sistema público de salud, como los radiofármacos contra receptores de somatostatina. Implementar sistemas que respondan de forma eficiente a las necesidades de los pacientes con TNEs, sumado a los notables avances terapéuticos 
en desarrollo, perimitirá generar un escenario promisorio para los pacientes con este tipo de neoplasias, cada vez más frecuentes en la práctica clínica.

\section{Referencias}

1. Lubrasch O. Ueber den primären Krebs des Ileum, nebst Bemerkungen über das gleichzeitige Vorkommen von Krebs und Tuberkolose. Virchows Arch. 1888; 111: 280-317.

2. Oberndorfer S. Karzinoide Tumoren des Dünndarms. Frankfurter Zeitschrift för Pathol. 1907; 1: 425-32.

3. Hallet J, Law CHL, Cukier M, Saskin R, Liu N, Singh $\mathrm{S}$. Exploring the rising incidence of neuroendocrine tumors: A population-based analysis of epidemiology, metastatic presentation, and outcomes. Cancer 2015; 121 (4): 589-97.

4. Dasari A, Shen C, Halperin D, Zhao B, Zhou S, Xu Y, et al. Trends in the Incidence, Prevalence, and Survival Outcomes in Patients With Neuroendocrine Tumors in the United States. JAMA Oncol. 2017; 3 (10): 1335-42.

5. Yao JC, Hassan M, Phan A, Dagohoy C, Leary C, Mares JE, et al. One hundred years after "carcinoid": Epidemiology of and prognostic factors for neuroendocrine tumors in 35,825 cases in the United States. J Clin Oncol. 2008; 26 (18): 3063-72.

6. Pinto MP, Muñoz-Medel M, Carrillo D, Retamal IN, Bravo ML, Valenzuela Y, et al. Chilean Registry for Neuroendocrine Tumors: A Latin American Perspective. Horm Cancer 2019; 10 (1): 3-10.

7. Kunz PL. Carcinoid and neuroendocrine tumors: Building on success. J Clin Oncol. 2015; 33 (16): 1855-63.

8. Klimstra DS. Pathology reporting of neuroendocrine tumors: Essential elements for accurate diagnosis, classification, and staging. Semin Oncol. 2013; 40 (1): 23-36.

9. Klimstra DS, Beltran H, Lilenbaum R, Bergsland E. The spectrum of neuroendocrine tumors: histologic classification, unique features and areas of overlap. Am Soc Clin Oncol Educ book. Am Soc Clin Oncol Meet. 2015; 92-103.

10. García-Carbonero R, Vilardell F, Jiménez-Fonseca P, González-Campora R, González E, Cuatrecasas M, et al. Guidelines for biomarker testing in gastroenteropancreatic neuroendocrine neoplasms: A national consensus of the Spanish Society of Pathology and the Spanish Society of Medical Oncology. Clin Transl Oncol. 2014; 16 (3): 243-56.

11. Lloyd RV, Osamura RY, Klöppel GRJ, editores. WHO Classification of Tumours of Endocrine Organs. WHO/
IARC Classification of Tumours. 4th ed. Lyon: IARC Press. 2017; 355.

12. Klöppel G, Rindi G, Perren A, Komminoth P, Klimstra DS. The ENETS and AJCC/UICC TNM classifications of the neuroendocrine tumors of the gastrointestinal tract and the pancreas: a statement. Virchows Arch. 2010; 456 (6): 595-7.

13. Klöppel G. Classification and pathology of gastroenteropancreatic neuroendocrine neoplasms. Endocr Relat Cancer 2011; 18 (1): 1-16.

14. Faggiano A, Ferolla P, Grimaldi F, Campana D, Manzoni M, Davì MV, et al. Natural history of gastro-entero-pancreatic and thoracic neuroendocrine tumors. Data from a large prospective and retrospective Italian epidemiological study: the NET management study. J Endocrinol Invest. 2012; 35 (9): 817-23.

15. Halperin DM, Shen C, Dasari A, Xu Y, Chu Y, Zhou S, et al. Frequency of carcinoid syndrome at neuroendocrine tumour diagnosis: a population-based study. Lancet Oncol. 2017;18 (4): 525-34.

16. Van der Horst-Schrivers AN, Wymenga AN, Links TP, Willemse PH, Kema IP, de Vries EG. Complications of midgut carcinoid tumors and carcinoid syndrome. Neuroendocrinology 2004; 80 (1): 28-32.

17. Hassan SA, Banchs J, Iliescu C, Dasari A, López-Mattei J, Yusuf SW. Carcinoid heart disease. Heart. 2017; 103 (19): 1488-95.

18. Van Essen M, Sundin A, Krenning EP, Kwekkeboom DJ. Neuroendocrine tumours: the role of imaging for diagnosis and therapy. Nat Rev Endocrinol. 2013; 10 (2): 102-14.

19. Sahani DV, Bonaffini PA, Fernández-Del Castillo C, Blake MA. Gastroenteropancreatic neuroendocrine tumors: Role of imaging in diagnosis and management. Radiology. 2013; 266 (1): 38-61.

20. Taupenot L, Harper KL, O'Connor DT. The Chromogranin-Secretogranin Family. N Engl J Med. 2003; 348 (12): 1134-49.

21. Modlin IM, Gustafsson BI, Moss SF, Pavel M, Tsolakis AV, Kidd M. Chromogranin A-Biological Function and Clinical Utility in Neuro Endocrine Tumor Disease. Ann Surg Oncol. 2010; 17 (9): 2427-43.

22. Marotta V, Nuzzo V, Ferrara T, Zuccoli A, Masone M, Nocerino L, et al. Limitations of Chromogranin A in clinical practice. Biomarkers. 2012; 17 (2): 186-91.

23. Bajetta E, Ferrari L, Martinetti A, Celio L, Procopio G, Artale S, et al. Chromogranin A, neuron specific enolase, carcinoembryonic antigen, and hydroxyindole acetic acid evaluation in patients with neuroendocrine tumors. Cancer 1999;86 (5): 858-65.

24. Feldman JM, O'dorisio TM. Role of neuropeptides and 
serotonin in the diagnosis of carcinoid tumors. Am J Med. 1986; 81 (6): 41-8.

25. Aluri V, Dillon JS. Biochemical Testing in Neuroendocrine Tumors. Endocrinol Metab Clin North Am. 2017; 46 (3): 669-77.

26. Khan MS, Kirkwood A, Tsigani T, García-Hernández J, Hartley JA, Caplin ME, et al. Circulating tumor cells as prognostic markers in neuroendocrine tumors. J Clin Oncol. 2013; 31 (3): 365-72.

27. Zatelli MC, Grossrubatscher EM, Guadagno E, Sciammarella C, Faggiano A, Colao A. Circulating tumor cells and miRNAs as prognostic markers in neuroendocrine neoplasms. Endocr Relat Cancer 2017; 24 (6): 223-37.

28. Kinross JM, Drymousis P, Jiménez B, Frilling A. Metabonomic profiling: a novel approach in neuroendocrine neoplasias. Surgery. 2013; 154 (6): 1185-92-3.

29. Modlin IM, Drozdov I, Alaimo D, Callahan S, Teixiera N, Bodei L, et al. A multianalyte PCR blood test outperforms single analyte ELISAs (chromogranin A, pancreastatin, neurokinin A) for neuroendocrine tumor detection. Endocr Relat Cancer 2014; 21 (4): 615-28.

30. Singh S, Asa SL, Dey C, Kennecke H, Laidley D, Law $\mathrm{C}$, et al. Diagnosis and management of gastrointestinal neuroendocrine tumors: An evidence-based Canadian consensus. Cancer Treat Rev. 2016; 47: 32-45.

31. Strosberg JR, Cheema A, Weber JM, Ghayouri M, Han G, Hodul PJ, et al. Relapse-Free Survival in Patients With Nonmetastatic, Surgically Resected Pancreatic Neuroendocrine Tumors. Ann Surg. 2012; 256 (2): 3215.

32. Rossi RE, Burroughs AK, Caplin ME. Liver transplantation for unresectable neuroendocrine tumor liver metastases. Ann Surg Oncol. 2014; 21 (7): 2398-405.

33. Sidéris L, Dubé P, Rinke A. Antitumor effects of somatostatin analogs in neuroendocrine tumors. Oncologist. 2012; 17 (6): 747-55.

34. Kvols LK, Moertel CG, O'Connell MJ, Schutt AJ, Rubin J, Hahn RG. Treatment of the malignant carcinoid syndrome. Evaluation of a long-acting somatostatin analogue. N Engl J Med. 1986; 315 (11): 663-6.

35. Rinke A, Wittenberg M, Schade-Brittinger C, Aminossadati B, Ronicke E, Gress TM, et al. Placebo-controlled, double-blind, prospective, randomized study on the effect of octreotide lar in the control of tumor growth in patients with metastatic neuroendocrine midgut tumors (PROMID): Results of long-term survival. Neuroendocrinology 2016; 104 (1): 26-32.

36. Caplin ME, Pavel M, wikła JB, Phan AT, Raderer M, Sedlá ková E, et al. Lanreotide in Metastatic Enteropancreatic Neuroendocrine Tumors. N Engl J Med. 2014; 371 (3): 224-33.
37. Wolin E, Jarzab B, Eriksson B, Walter T, Toumpanakis C, Morse MA, et al. Phase III study of pasireotide long-acting release in patients with metastatic neuroendocrine tumors and carcinoid symptoms refractory to available somatostatin analogues. Drug Des Devel Ther. 2015; 9 (9): 5075-86.

38. Anthony LB, Woltering EA, Espenan GD, Cronin MD, Maloney TJ, McCarthy KE. Indium-111-pentetreotide prolongs survival in gastroenteropancreatic malignancies. SeminNucl Med. 2002; 32 (2): 123-32.

39. Strosberg J, El-Haddad G, Wolin E, Hendifar A, Yao J, Chasen B, et al. Phase 3 Trial of 177 Lu-Dotatate for Midgut Neuroendocrine Tumors. N Engl J Med. 2017; 376 (2): 125-35.

40. Tworowska I, Delpassand ES, Bolek L, Shanoon F, Sgouros G, Frey E, et al. Targeted Alpha-emitter Therapy of Neuroendocrine Tumors using 212Pb-octreotate (AlphaMedix TM). J Med Imaging and Rad Sci. 2019; 50 (4): 105-6.

41. Pavel ME, Baudin E, Öberg KE, Hainsworth JD, Voi M, Rouyrre N, et al. Efficacy of everolimus plus octreotide LAR in patients with advanced neuroendocrine tumor and carcinoid syndrome: final overall survival from the randomized, placebo-controlled phase 3 RADIANT-2 study. Ann Oncol. 2017; 28 (7): 1569-75.

42. Yao JC, Shah MH, Ito T, Bohas CL, Wolin EM, Van Cutsem E, et al. Everolimus for advanced pancreatic neuroendocrine tumors. N Engl J Med. 2011; 364 (6): 514-23.

43. Yao JC, Fazio N, Singh S, Buzzoni R, Carnaghi C, Wolin E, et al. Everolimus for the treatment of advanced, non-functional neuroendocrine tumours of the lung or gastrointestinal tract (RADIANT-4): a randomised, placebo-controlled, phase 3 study. Lancet. 2016; 387 (10022): 968-77.

44. Raymond E, Dahan L, Raoul J-L, Bang Y-J, Borbath I, Lombard-Bohas C, et al. Sunitinib malate for the treatment of pancreatic neuroendocrine tumors. N Engl J Med. 2011; 364 (6): 501-13.

45. Kulke MH, Stuart K, Enzinger PC, Ryan DP, Clark JW, Muzikansky A, et al. Phase II study of temozolomide and thalidomide in patients with metastatic neuroendocrine tumors. i. 2006; 24 (3): 401-6.

46. Strosberg JR, Fine RL, Choi J, Nasir A, Coppola D, Chen $\mathrm{D}-\mathrm{T}$, et al. First-line chemotherapy with capecitabine and temozolomide in patients with metastatic pancreatic endocrine carcinomas. Cancer 2011; 117 (2): 268-75.

47. Faure M, Niccoli P, Autret A, Cavaglione G, Mineur L, Raoul J-L. Systemic chemotherapy with FOLFOX in metastatic grade $1 / 2$ neuroendocrine cancer. Mol Clin Oncol. 2017; 6 (1): 44-8. 
48. Pavel M, O'Toole D, Costa F, Capdevila J, Gross D, Kianmanesh R, et al. ENETS Consensus Guidelines Update for the Management of Distant Metastatic Disease of Intestinal, Pancreatic, Bronchial Neuroendocrine Neoplasms (NEN) and NEN of Unknown Primary Site. Neuroendocrinology. 2016; 103 (2): 172-85.

49. Mitry E, Baudin E, Ducreux M, Sabourin JC, Rufié P, Aparicio T, et al. Treatment of poorly differentiated neuroendocrine tumours with etoposide and cisplatin. Br J Cancer. 1999; 81 (8): 1351-5.

50. Strosberg JR, Coppola D, Klimstra DS, Phan AT, Kulke $\mathrm{MH}$, Wiseman GA, et al. The NANETS Consensus Guidelines for the Diagnosis and Management of Poorly Differentiated (High-Grade) Extrapulmonary Neuroendocrine Carcinomas. Pancreas 2010; 39 (6): 799-800.

51. Sorbye H, Welin S, Langer SW, Vestermark LW, Holt $\mathrm{N}$, Osterlund $\mathrm{P}$, et al. Predictive and prognostic factors for treatment and survival in 305 patients with advanced gastrointestinal neuroendocrine carcinoma (WHO G3): the NORDIC NEC study. Ann Oncol. 2013;24 (1): 15260.

52. Molina-Cerrillo J, Alonso-Gordoa T, Martínez-Sáez O, Grande E. Inhibition of Peripheral Synthesis of Serotonin as a New Target in Neuroendocrine Tumors. Oncologist. 2016; 21 (6): 701-7.

53. Carroll TB, Peppard WJ, Herrmann DJ, Javorsky BR, Wang TS, Patel H, et al. Continuous Etomidate Infusion for the Management of Severe Cushing Syndrome: Validation of a Standard Protocol. J Endocr Soc. 2018; 3 (1): 1-12.

54. Fleseriu M, Castinetti F. Updates on the role of adrenal steroidogenesis inhibitors in Cushing's syndrome: a focus on novel therapies. Pituitary 2016; 19 (6): 643-53.

55. Eriksson B, Klöppel G, Krenning E, Ahlman H, Plöckinger U, Wiedenmann B, et al. Consensus guidelines for the management of patients with digestive neuroendocrine tumors - Well-differentiated jejunal-ileal tumor/ carcinoma. Neuroendocrinology 2007; 87 (1): 8-19.

56. Russolillo N, Vigano' L, Razzore P, Langella S, Motta M, Bertuzzo F, et al. Survival prognostic factors of gastro-enteric-pancreatic neuroendocrine tumors after primary tumor resection in a single tertiary center: Comparison of gastro-enteric and pancreatic locations. Eur J Surg Oncol. 2015; 41 (6): 751-7.

57. Ducreux M. Carcinoid syndrome in neuroendocrine tumors: a prognostic effect? Lancet Oncol. 2017; 18 (4): 426-8.

58. Oberg K, Modlin IM, De Herder W, Pavel M, Klimstra $\mathrm{D}$, Frilling A, et al. Consensus on biomarkers for neuroendocrine tumour disease. Lancet Oncol. 2015; 16 (9): 435-46.

59. García-Carbonero R, Capdevila J, Crespo-Herrero G, Díaz-Pérez JA, Martínez del Prado MP, Alonso Orduña $\mathrm{V}$, et al. Incidence, patterns of care and prognostic factors for outcome of gastroenteropancreatic neuroendocrine tumors (GEP-NETs): Results from the National Cancer Registry of Spain (RGETNE). Ann Oncol. 2010; 21 (9): 1794-803.

60. Lepage C, Rachet B, Coleman MP. Survival From Malignant Digestive Endocrine Tumors in England and Wales: A Population-Based Study. Gastroenterology 2007; 132 (3): 899-904. 